\title{
Ultrasonography of the Salivary Gland in Primary Sjögren Syndrome: Usefulness to Phenotype the Patients
}

\author{
Valerie Devauchelle-Pensec ${ }^{1}$
}

Major salivary gland ultrasonography (SGUS) is widely used to diagnose primary Sjögren syndrome (pSS) in a large majority of individuals as well as in specific situations, such as when lymphoma is suspected ${ }^{1,2,3}$. It is a powerful and noninvasive procedure that provides a global image of the parenchymal lesions of the major salivary gland and clearly reflects the effect of the disease on the target tissue ${ }^{4}$. Some different scoring systems exist, all of which are based on parenchymal inhomogeneity of the parotid and submandibular glands. In this issue of The Journal of Rheumatology, Mossel, et al included consecutive primary Sjögren syndrome (pSS) outpatients in a registry of patients called the RESULT cohort; they described a phenotype associated with an elevated US parenchymal score 5 . Based on the Hocevar scoring system, an elevated US score was associated with more severe disease, higher European League Against Rheumatism (EULAR) Sjögren's Syndrome Disease Activity Index (ESSDAI) score, higher levels of IgG and rheumatoid factor, and more frequent positive parotid gland biopsy, anti-SSA/SSB antibodies, abnormal unstimulated whole saliva, and ocular staining score than SGUS-negative patients. In contrast, patients with an elevated ultrasound (US) score had less fatigue and pain than those without US lesions.

Despite some bias (longer disease duration in the elevated US score group and the absence of similar results in other cohorts), it is believed that SGUS may help to delineate a specific phenotype of pSS, echoing other results under investigation. Tarn, et al ${ }^{6}$ described 4 different phenotypes of patients based on patient-reported outcomes and symptoms; within 2 groups, the predominance

${ }^{I} V$. Devauchelle-Pensec, $M D, P h D$, Rheumatologist, Rheumatology Department, INSERM UMR 1227, Cavale Blanche Hospital and Brest Occidentale University, Brest, France.

The author declares no conflict of interest concerning this manuscript. Address correspondence to Dr. V. Devauchelle-Pensec, Service de rhumatologie, CHRU Cavale Blanche, Boulevard Tanguy Prigent, 29200, Brest, France.

Email:valerie.devauchelle-pensec@chu-brest.fr. of fatigue was underlined by different biological processes. This led to an ongoing European project named Stratified Medicine in Primary Sjögren's Syndrome. This also highlights the fact that fatigue seems to be a complex symptom, probably driven by genetics and heterogeneous components. Another project ${ }^{7}$ aimed to reclassify systemic autoimmune diseases and to describe different genotypes/phenotypes of patients, including those with SS (PRECISESADS; ClinicalTrials.gov: NCT02890121). Therefore, the SGUS score could be used to define subtypes of patients when combined with clinical symptoms.

Mossel, et al also noted the high frequency of SGUS abnormalities in patients with $\mathrm{pSS}^{5}$. A total of $79 \%(136 / 172)$ of their patients had parenchymal inhomogeneity, with a mean disease duration of $8.0(4.0-13.0)$ years. This highlights the interest in the US procedure in classifying pSS. We and others have also demonstrated that SGUS has high accuracy for diagnosing pSS with a recent disease duration of less than 5 years ${ }^{8,9}$. It is also used in the ACR/EULAR 2016 classification criteria ${ }^{10}$. The addition of SGUS as a minor item increases the sensitivity in the whole cohort from $90.2 \%$ to $95.6 \%$ according to expert opinions and increases the sensitivity in a cohort of patients suspected of having Sjörgren syndrome from $87 \%$ to $93 \%$. Therefore, SGUS has good diagnostic and classification accuracy for pSS.

Finally, in their study, Mossel, et al showed that an elevated US score was associated with a longer disease duration ${ }^{5}$. Only 36 patients had a low US score, with a mean disease duration of 5.0 (3.0-8.8) years. However, in the group of patients with abnormal US scores, the disease duration was similar between those with medium $(n=67)$ and high $(n=69)$ US scores. This trend suggests that SS progresses slowly, leading to parenchymal destruction. However, this point remains questionable. The question is whether this high score is linked to the duration of the disease or to its overall activity, as stated by the authors. It is difficult in the absence of a real prospective cohort to describe the pathological progression of the parenchyma, and relevant data are lacking; however, we know that scoring SGUS lesions using

\section{See Phenotyping pSS with US, page 717}


a global score with a 2-year interval did not demonstrate strong evolution of the score ${ }^{11}$. On US, it is believed that anechoic or hypoechoic foci are related to inflammation inside the gland. Fibrosis is defined as the presence of hyperechoic bands in fibrotic tissue indistinguishable from adjacent tissue. Another stage related to terminal modification of the parenchyma is the presence of fatty lesions that are homogeneously hyperechoic. However, we still need to explore the biopathological process leading to irreversible lesions, as well as the close relationship between inflammation and fibrosis inside the parenchyma. More recent therapeutic trials have demonstrated that we can interfere with the parenchymal process, and the results of SGUS suggest for the first time that in some cases, the parenchyma can be partially restored by immunotherapy ${ }^{12,13}$.

Therefore, SGUS is a simple and very informative procedure that is not only helpful at the patient level for the diagnosis and classification of SS but also serves as a window for an autoimmunity target that can be combined with other data to delineate the phenotypes of patients and endpoints for clinical trials.

\section{REFERENCES}

1. Baer A, Grader-Beck T, Antiochos B, Birnbaum J, Fradin J. Ultrasound-guided biopsy of suspected salivary gland lymphoma in Sjögren's syndrome. Arthritis Care Res 2020 Apr 5 (E-pub ahead of print).

2. Jonsson MV, Baldini C. Major salivary gland ultrasonography in the diagnosis of Sjogren's syndrome: a place in the diagnostic criteria? Rheum Dis Clin North Am 2016;4:501-17.

3. Devauchelle-Pensec V, Zabotti A, Carvajal-Alegria G, Filipovic N, Jousse-Joulin S, De Vita S. Salivary gland ultrasonography in primary Sjogren's syndrome: opportunities and challenges. Rheumatology 2019 Mar 19 (E-pub ahead of print).

4. Mossel E, Delli K, van Nimwegen JF, Stel AJ, Haacke EA, Kroese FG, et al. The parotid gland connection: ultrasound and biopsies in primary Sjogren's syndrome. Ann Rheum Dis 2018;77:e38.

5. Mossel E, van Nimwegen JF, Stel AJ, Wijnsma RF, Delli K, van Zuiden GS, et al. Clinical phenotyping of primary Sjögren patients using salivary gland ultrasonography: data from the RESULT cohort. J Rheumatol 2021;48;717-27.
6. Tarn JR, Howard-Tripp N, Lendrem DW, Mariette X, Saraux A, Devauchelle-Pensec V, et al; French ASSESS cohort; UK Primary Sjögren's Syndrome Registry. Symptom-based stratification of patients with primary Sjögren's syndrome: multi-dimensional characterisation of international observational cohorts and reanalyses of randomised clinical trials. Lancet Rheumatol 2019;1:e85-94.

7. Beretta L, Barturen G, Vigone B, Bellocchi C, Hunzelmann N, De Langhe E, et al; PRECISESADS SSc substudy group; PRECISESADS Flow Cytometry study group. Genome-wide whole blood transcriptome profiling in a large European cohort of systemic sclerosis patients. Ann Rheum Dis 2020;79:1218-26.

8. Cornec D, Jousse-Joulin S, Marhadour T, Pers JO, Boisramé-Gastrin S, Renaudineau Y, et al. Salivary gland ultrasonography improves the diagnostic performance of the 2012 American College of Rheumatology classification criteria for Sjogren's syndrome. Rheumatology 2014;53:1604-7.

9. Le Goff M, Cornec D, Jousse-Joulin S, Guellec D, Costa S, Marhadour T, et al. Comparison of 2002 AECG and 2016 ACR/ EULAR classification criteria and added value of salivary gland ultrasonography in a patient cohort with suspected primary Sjogren's syndrome. Arthritis Res Ther 2017;19:269.

10. Jousse-Joulin S, Gatineau F, Baldini C, Baer A, Barone F, Bootsma $\mathrm{H}$, et al. Weight of salivary gland ultrasonography compared to other items of the 2016 ACR/EULAR classification criteria for primary Sjogren's syndrome. J Intern Med 2020;287:180-8.

11. Gazeau, P, Cornec D, Jousse-Joulin S, Guellec D, Saraux A, Devauchelle-Pensec V. Time-course of ultrasound abnormalities of major salivary glands in suspected Sjogren's syndrome. Joint Bone Spine 2018;85:227-32.

12. Jousse-Joulin S, Devauchelle-Pensec V, Cornec D, Marhadour T, Bressollette L, Gestin S, et al. Brief report: ultrasonographic assessment of salivary gland response to rituximab in primary Sjogren's syndrome. Arthritis Rheumatol 2015;67:1623-8.

13. Fisher BA, Everett CC, Rout J, O’Dwyer JL, Emery P, Pitzalis C, et al. Effect of rituximab on a salivary gland ultrasound score in primary Sjogren's syndrome: results of the TRACTISS randomised double-blind multicentre substudy. Ann Rheum Dis 2018;77:412-6. 